\title{
Schlafkrankheit bei deutschen Tropenreisenden
}

\author{
Th. Löscher. H. D. Nothdurft. H. Taelman. M. Boogaerts, M. Omar und F. von Sonnenburg \\ Abteilung für Infektions- und Tropenmedizin der Universität München. Institut für Tropenmedizin, Antwerpen.
} und Centre médico-social Kanombe, Kigali. Ruanda

\begin{abstract}
Zwei deutsche Tropenreisende erkrankten nach einer zweitägigen Safari im Akagera-Nationalpark in Ruanda an ostafrikanischer Trypanosomiasis (Schlafkrankheit). Leitsymptome waren Fieber. Lymphadenopathie und eine typische Primärläsion (Trypanosomenschanker). Die Diagnose konnte durch den Nachweis von Trypanosomen im peripheren Blut gesichert werden. Das Zentralnervensystem war in beiden Fällen nicht beteiligt. Unter dem Einfluß einer Therapie mit Suramin, $1 \mathrm{~g}$ pro Woche intravenös über 6 Wochen, bildeten sich die Symptome und die Parasitämie rasch zurück. Nach der Zahl der seit 1970 berichteten Fälle ergibt sich für deutsche Tropenreisende ein Infektionsrisiko von 0.3 pro 100000 . Aufgrund der teilweise erheblichen Wiederzunahme der Schlafkrankheit in einigen afrikanischen Ländern kann mit einer Zunahme des Infektionsrisikos auch für Touristen gerechnet werden.

\section{African trypanosomiasis in German visitors to Ruanda}

A brother and sister the latter having been resident in Ruanda for three years) fell ill with African trypanosomiasis (sleeping sickness) after a two-day safari in the Akagera National Park. Cardinal symptoms were fever, lymphadenopathy and the typical primary lesion (trypanosomal chancre). The diagnosis was confirmed by demonstrating trypanosomes in the peripheral blood. There was no CNS involvement in either case. Administration of suramin, $1 \mathrm{~g}$ weekly intravenously for six weeks, quickly brought about regression of the symptoms and the parasitaemia. According to the number of cases reported since 1970. the risk for German travellers to certain African areas of contracting trypanosomiasis is about 0.3 per 100000 . Since in Africa the incidence of the disease is increasing. in some parts considerably. one must reckon with an increasing risk for tourists.
\end{abstract}

Die während der letzten drei Jahrzehnte ständig zunehmenden Fernreisen in tropische und subtropische Gebiete sind mit dem erhöhten Risiko verbunden, zum Teil schwerwiegende Gesundheitsstörungen zu erleiden (14). Im Vordergrund der möglicherweise lebensbedrohlichen Erkrankungen steht die Malaria tropica (9), deren zunehmende Häufigkeit in vielen Ländern nicht nur auf Resistenzentwicklungen. sondern auch auf ökologischen und ökonomischen Faktoren, wie Bewässerungsprojekten. Fehlen der Mittel zur Bekämpfung. Unterbrechung von Bekämpfungsprogrammen aus politischen Gründen, beruht. Die afrikanische Trypanosomiasis (Schlafkrankheit) stellt eine weitere durch Protozoen verursachte Tropenkrankheit dar.

Dtsch. med. Wschr. 114 (1989), 1203-1206

(4) Georg Thieme Verlag Stuttgart- New York die aufgrund ähnlicher Faktoren in den letzten Jahren ebenfalls in einigen Endemiegebieten teilweise drastisch zugenommen hat (18). Das Infektionsrisiko für Reisende aus nicht endemischen Gebieten ist im Vergleich mit der Malaria bislang jedoch äußerst gering $(14,17)$. Andererseits handelt es sich bei der Trypanosomiasis um eine schwerwiegende, unbehandelt mit wenigen Ausnahmen zum Tode führende Erkrankung. Im folgenden berichten wir über zwei deutsche Tropenreisende, die an ostafrikanischer Schlafkrankheit erkrankten.

\section{Kasuistik}

Anamnese: Eine 39 jährige deutsche Entwicklungshelferin, die seit 3 Jahren in Kigali, Ruanda, als Lehrerin arbeitete, wurde im Dezember 1987 von ihrem 51 jahrigen Bruder aus Deutschland besucht. Finf Tage nach einer gemeinsamen zweitägigen Safari im Akagera-Nationalpark entwickelte sich beim Bruder (fall 1) eine gering dolente. 


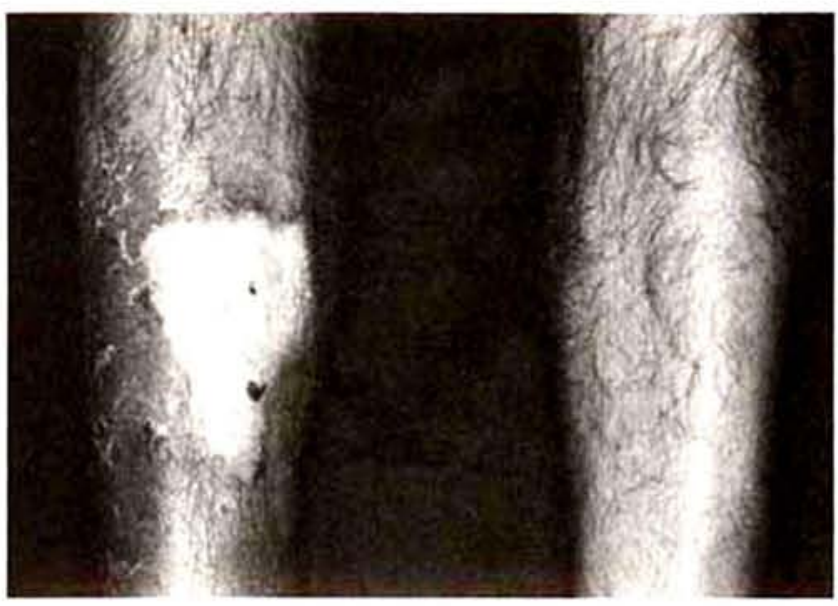

Abb. 1 Pnmarlasion (Trypanosomenschanker) bei Fall 1

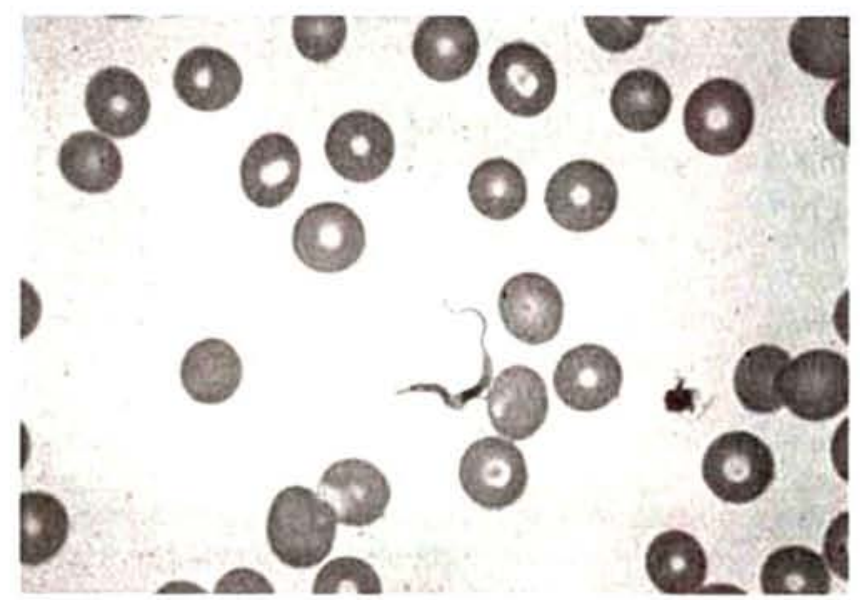

Abb. 2 Trypanosoma brucei thodesiense im peripheren Blutausstrich

gerötete Schwellung uber der rechten Tibia, die er zunachst nicht beachtete. Drei Tage spater kam es zu fieber bis $39.5^{\circ} \mathrm{C}$ ohne Schuttelfrost mit Kopfschmerzen, Myalgien, allgemeiner Schwache und einer indolenten l.ymphknotenvergroßerung in der rechten leiste. Die prätibiale Schwellung hatte sich weiter vergrößert und zeigte zentral eine $3-4 \mathrm{~mm}$ große schwärzliche Nekrose (Abbildung 11. Am folgenden Tag suchte der Patient die Ambulanz des Centre médico-social Kanombe in Kigali auf. Dort wurde ein Blutausstrich angefertigt, in dem sich vereinzelt Trypanosomen fanden (Abbildung 2).

Bei seiner Schwester (1)all $21 \mathrm{kam}$ es am selben Tag erstmals zu Fieber und Allgemeinsymptomen. Am nächsten Tag wurden im sdicken Tropfen $\alpha$ ebenfalls vereinzelt Trypanosomen gefunden. Bei beiden Patienten wurde am Tag der Diagnose eine Therapie mit Suramin $11 \mathrm{~g}$ langsam intravenos nach einer Testdosis von $100 \mathrm{mg}$ begonnen. Ein und zwei Tage spater erfolgte der Ruckflug unter ärtlicher Oberwachung zur weiteren Diagnostik und Therapic

Befunde. Die klinischen und klinisch-chemischen Befunde bei der Aufnabme in unserer Klinik sind in Tabelle 1 zusammengefaßt. Auch bei fall 2 konnte bei der klinischen Untersuchung eine Primärläsion entdeckt werden. die allerdingts weit weniger ausgeprägt und von der Patientin bislang nicht bemerkt worden war: Am rechten Unterschenkel fand sich dorsal eine gering indurierte und erhabene schmerz. lose Rotung von etwa $2.5 \mathrm{~cm}$ Durchmesser ohne Nekrosen und ohne erkennbare Stichstelle. Bei beiden Patienten waren zer.
Tab.1 Kinische und Kinisch-chemische Befunde bei zwei Patienten mit afrikanischer Trypanosomiasis (Schlafkrankheit)

\begin{tabular}{|c|c|c|}
\hline Befunde & Fall 1 & Fall 2 \\
\hline Fieber & $38,8^{\circ} \mathrm{C}$ & $39.0^{\circ} \mathrm{C}$ \\
\hline Primatlasion & ++ & + \\
\hline Lymphadenopathie & + & ++ \\
\hline Splenomegalie & + & - \\
\hline Leukozyten/ul & 2000 & 4400 \\
\hline $\lg M(g / \mid)$ & 7.1 & 4.9 \\
\hline \multicolumn{3}{|l|}{ Serologe } \\
\hline \multicolumn{3}{|l|}{ IFAT IBG } \\
\hline \multirow{2}{*}{ ELISA } & $1: 128$ & $1: 256$ \\
\hline & $>100^{\circ}$ & $62^{\circ}$ \\
\hline IHA & $1: 32$ & $1: 16$ \\
\hline \multicolumn{3}{|l|}{ Liquor } \\
\hline \multicolumn{3}{|l|}{ Zellzahl, GesamterweiB, IgG } \\
\hline \multirow{2}{*}{$\begin{array}{ll}\text { IFAT IgG } \\
\quad \mathrm{IgM}\end{array}$} & negativ & negativ \\
\hline & negativ & negativ \\
\hline
\end{tabular}

IFAT = Immunfluoreszenz Antikorpertest (Antigen: Trypanosoma bruce brucei), ELISA - Enzyme Linked Immunosorbent Assay (Antigen: Typanosoma brucei brucei). IHA = indirekte Hamaggiutination (Cellognost Trypanosomasis Behring Antigen: Trypanosoma brucei gambiense)

- Vielfaches der Normalaktivitat

vikal und inguinal vergrößerte druckdolente Lymphknoten tastbar. Bei Fall 1 bestanden zudem ein Ikterus und eine tastbare Milzvergrößerung: die Röntgenaufnahme des Thorax wies einen kleinen PleuraerguB links auf. Im Elektrokardio. gramm fielen Repolarisationsstörungen links präkordial mit muldenformiger ST-Senkung und T-Abflachung auf.

Die Liquorbefunde bei beiden Patienten waren völlig normal: weder Trypanosomen noch autochthone Antikörper waren im l.iquor nachweisbar.

Therapie und Verlauf. Die Behandlung mit Suramin wurde in einer Dosierung von $1 \mathrm{~g}$ einmal wöchentlich intravenos bis zu einer Gesamtdosis von $6 \mathrm{~g}$ fortgefuhrt. Bei Fall 1 waren bereits ab dem zweiten Tag. bei Fall 2 ab dem vierten Tag nach der ersten Suramin-Injektion auch mittels Konzentrationsmethoden (Mikrokapillarmethode, lonenaustausch-Chromatographie nach lanham) im Blut keine Trypanosomen mehr nachweisbar. Beide Patienten waren nach der «weiten Behandlungswoche beschwerdefrei. Die pathologi. schen Befunde bildeten sich innerhalb von drei Wochen vollständig zurück. Als mögliche Nebenwirkung von Suramin kam es bei fall 1 zu einer reversiblen Proteinurie und Glucosurie.

Bei Kontrolluntersuchungen 1,3 und 6 Monate nach der Therapie lagen bei beiden Patienten sämtliche Befunde im Normbereich.

\section{Diskussion}

Dic afrikanische Trypanosomiasis (Schlafkrankheit) wird durch zwei eng verwandte, morphologisch nicht zu unterscheidende Unterarten der Trypanosoma-brucei-Gruppe verursacht: T. brucei gambiense (Frreger der westafrikanischen Schlafkrankheit) und $\mathrm{T}$. brucei rhodesiense (Erreger der ostafrikanischen Schlafkrankheit). Die Ubertragung erfolgt durch den Stich infizierter Tsetse-Fliegen (Glossinen), selten diaplazentar, über Bluttrans- 


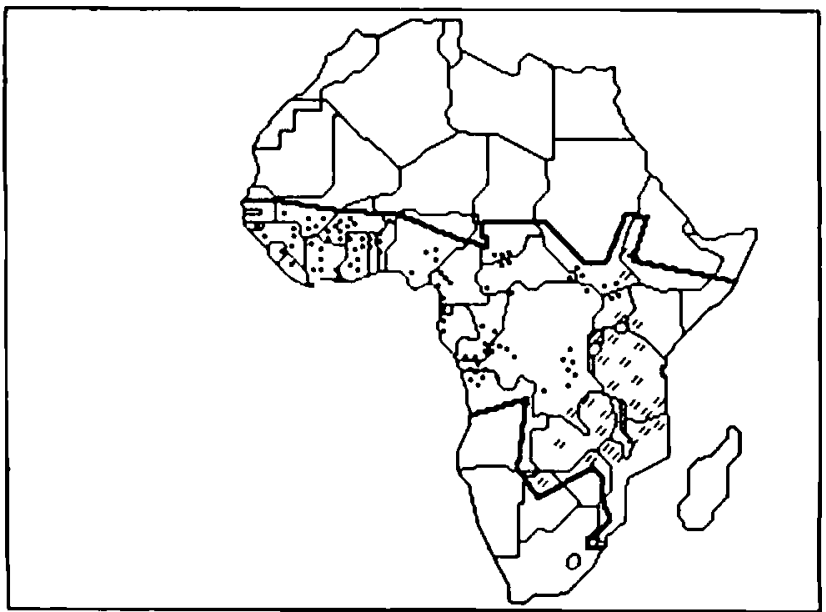

Abb. 3 Hauplendemiegebiete der atrikanischen Tryanosomiasis (Schlaikrankheit) nach Sturchlet (15) - Trypanosoma bruces gambiense. Trypanosoma brucei rhodesiense. - Tsetse-Gurel.

fusionen oder als Laborinfektion. Innerhalb des sogenannten Tsetse-(iürtels zwischen dem 15. nördlichen und dem 20. südlichen Breitengrad bestehen meist fokale Verbreitungsgebiete (Abbildung 3 ) $\mathrm{mit}$ jährlichen Inzidenzraten zwischen 1 und 30 fällen pro 100000 linwohner (15. 17). Mindestens 50 Millionen Menschen in ländlichen Gebieten von 32 Staaten sind derzeit potentiell exponiert (17. 18). In neun Staaten mit regelmäßigen Meldungen ergab sich von 1976 bis 1983 eine Zunahme der gemeldeten Neuer. krankungen um über das Doppelte von etwa 5000 auf rund 12000 pro Jahr. Dic für die meisten I.änder nur unvollständig verfügbaren Informationen ergaben von 1983 bis 1986 eine noch höhere Zunahmerate. Allein in /aire wurden 1986 über 10000 Fälle neu diagnostiziert (18).

Bei unseren beiden Patienten kommt aus epidemiologischen Grïnden nur $\mathrm{T}$. brucei rhode. siense als Firreger in Frage $(1,9,17)$. Der AkageraNationalpark in Ruanda ist als Endemiegebiet bekannt; von 1969 bis 1979 wurden von dort jedoch lediglich 34 Neuerkrankungen gemeldet (1). Im letzten bei einem deutschen Tropenreisenden diagnostizierten Fall von Trypanosomiasis war es 1985 ebenfalls dort zu einer Infektion gekommen (llartmann: persönliche Mitteilung). Einer Infektion mit $T$. brucei rhodesiense entspricht auch der akute klinische Verlauf mit einer in beiden fällen extrem kurzen Inkubationszeit. Die diagnostisch zum Teil wegweisende Primärläsion (sogenannter Trypanosomenschanker) tritt bei Weißen wesentlich häufiger auf als bei Afrikanern. Sic ist bei Europäern in etwa $50 \%$ der Fälle nachweisbar oder erinnerlich (3). Fieber. Lymphadenopathie und Splenomegalie sind im Initialstadium fast regelmäßig vorhanden. Die vollständig reversiblen EKG-Veränderungen bei Fall 1 sind als Hinweis auf eine ebenfalls häufig aufretende Myokarditis $\% u$ werten (6).
Entscheidend fur Therapie und Prognose ist die früheitige Diagnose in ersten, febrilglandulären Stadium. möglichst vor Beginn einer Invasion der Frreger ins Zentralnervensytem. Ziel der Diagnostik ist der direkte Parasitennachweis in Blut, L.iquor. Primärläsion und (oder) lymphknotenpunktat sowic eventuell auch im Knochenmark. Häufig sind hierzu Anreicherungsmethoden erforderlich (dicker Tropfen. Mikrokapillarmethode, Ionenaustausch-(hromatographie nach lanham). Der Nachweis spezifischer Antikörper und der meist stark erhöhten Gesamt-IgM-Serumkonzentration sollte Anlaß für eine Intensivierung der parasitologischen Diagnostik sein. Mittel der Wahl im ersten Stadium der ustafrikanischen Trypanosomiasis ist Suramin. Resistenzen sind nicht gesichert. Es kann jedoch innerhalb von 1-2 Jahren zu Rezidiven kommen. die meist wohl auf einer bereits erfolgten, aber nicht erfaßten Invasion des Zentralnervensystems beruhen.

Möglicherweise spielen auch intrazellulär gelegene Trypanosomen eine Rolle, die in ependymalen Zellen entlang der (iefäße des Plexus choroideus gefunden wurden (10). Zum Ausschluß einer ZNS-Beteiligung sollte in jedem $F$ all $3-4$ lage nach Therapiebeginn eine Liquoruntersuchung erfolgen. Im zweiten enzephalitischen Stadium standen bisher nur hochtoxische Substanzen wie Arsenpräparate (Melarsoprol) für eine wirksame Therapie zur Verfügung. Neuerdings zeigte das relativ atoxische Difluormethylornithin (DI:MO, Lnomithin) eine gute Wirksamkeit auch im enzephalitischen Siadium von Trypanosoma-brucei-gambiense-Infektionen (12). Eine Chemoprophylaxe mit Suramin oder dem nur gegen T. brucei gambiense wirksamen Pentamidin ist zwar möglich, sie wird heute aber abgelehnt wegen ihrer potentiellen Toxizität und der Möglichkeit. daß bei subkurativen Spiegeln und (oder) reduzierter limpfindlichkeit riniger Trypanosomenstämme aktuelle klinische Symptome unterdrückt werden und die unbemerkte Entwicklung von Spitstadien begünstigt wird (17). \%ur individuellen Prophylaxe bleibt im wesentlichen die Moidung der meist bekannten Isetse-(iebicte. Insektenschut\%mittel und das Tragen möglichst weitgehend hautbedekkender Kleidung sind nur teilweise wirksam gegren die Stiche der tagaktiven Glossinen.

Das Risiko für Reisende ist im Vergleich mit anderen l'ropenkrankheiten wie der Malaria als sehr gering cinzuschäzen $(14)$. Von Duggan und llutchinson (3) wurden bis 1963 insgesam 109) Fälle bei Europäern aus der Literatur zusammengestellt. Bei amerikanischen Tropenreisonden traten von 1967 bis $198714 \mathrm{l}$ älle ostafrikanischer Schlafkrankheit auf (2). Bereits zwei Jahre nach der Frstbeschreibung der afrikanischen Trypanosomiasis durch Dutton wurde 1904 der erste bei einem deutschen Tropenreisenden diagnostizierte lall von Gïnther und Weber (4) mitgeteilt. line Auswertung 
Tab. 2 Trypanosomiasis bei deutschen Tropenreisenden seit 1958 durch Trypanosoma bruce: rhodesiense (R). Trypanosoma brucei gambiense (G) und ohne parasitologische Sicherung (S. Winisch und serologisch wahrscheinlich)

\begin{tabular}{|c|c|c|c|c|}
\hline Jaht & $\begin{array}{l}\text { Aufent: } \\
\text { haitsort: }\end{array}$ & Erreger & Bemerkungen & Literatur \\
\hline 1958 & Lberia & G & Bauarbeiter & Mohr (8) \\
\hline 1970 & Zaire & $\mathrm{R}$ & Safantourist & $\begin{array}{l}\text { Seitz. Hofler: } \\
\text { personliche Mitteilung }\end{array}$ \\
\hline 1974 & Kamerun & S & Safaritourist & Werner. Stickl (16) \\
\hline 1976 & Botswana & $\mathrm{R}$ & Zoologe & Recht et al (11) \\
\hline 1976 & Sambia & S & Tounstin & $\begin{array}{l}\text { Eichenlaub. Pohle: } \\
\text { personliche Mitteilung }\end{array}$ \\
\hline 1978 & Sudan & R & Jager & Knuttgen (7) \\
\hline 1983 & Tansania & R & Jager & Hartmann, Racz (5) \\
\hline 1985 & Ruanda & R & Safaritouristin & $\begin{array}{l}\text { Hartmann: } \\
\text { personliche Mitteliung }\end{array}$ \\
\hline
\end{tabular}

der Literatur sowie eine Umfrage bei allen deutschsprachigen Einrichtungen, welche die parasitologische und (oder) serologische Diagnostik der Schlafkrankheit durchführen, ergab insgesamt sechs parasitologisch gesicherte Fälle bei deutschen Afrikareisenden während der letzten 30 Jahre (Tabelle 2). In zwei weiteren Fällen wurde die Diagnose aufgrund der klinischen und serologischen Befunde gestellt. ohne daß ein direkter Nachweis der Trypanosomen gelang.

Unter Berücksichtigung der Reisefrequenz in Länder mit Endemiegebieten (13) ergibt sich für deutsche Tropenreisende ein rechnerisches Risiko von 0.3 pro 100000 in der Zeit von 1970 bis 1988. Das Risiko einer Malariaerkrankung. unabhängig von Art und Durchführung einer Chemoprophylaxe, liegt bei Reisen in diese Länder derzeit bei $50-300$ auf $100000(9.13)$. Allerdings ist anzunehmen. daß das Risiko einer Schlafkrankheit bei Aufenthalten in den fokalen Endemiegebieten und bei besonderer Exposition (Jäger. Safaris. Entwicklungshelfer, Missionare) deutlich höher ist. Zudem besteht die Möglichkeit einer Dunkelziffer nicht diagnostizierter Fälle. Aufgrund der teilweise erheblichen Wiederzunahme der Schlafkrankheit in einigen afrikanischen Ländern muß mit einer Zunahme des Infektionsrisikos auch bei Touristen gerechnet werden.

\section{Literatur}

1 Burke. J.: Trypanosomiase. In Meheus. A.. S. Butera. W Eylenbosch. G. Gotera, M. Kinits, 1. Musofili (Hirsg.): Santé et Maladies au Rwanda (Administration Générale de la Cooperation au Développement: Bruxelles, 1982), 448.

2 Bryan, R. T., H. Waskin, F. Richards, T. Bailey, D. Juranek: African trypanosomiasis in American travelers. A 20 year review. Conference of International Travel Medicine, Zürich (1988).

3 Duggan. A. J., M. P. Hutchinson: Sleeping sickness in Europeans. A review of 109 cases. J. trop. Med. Hyg. 69 (1966), 124

4 Günther, H., R. Weber: Ein Fall von Trypanosomenkrankheit beim Menschen. Münch. med. Wschr. 51 (1904), 1044.

5 Hartmann. G.. P. Racz: Infektion mit Trypanosoma rhodesiense nach Tansania-Aufenthalt. 11. Symposion der Deutschen Gesellschaft für Infektiologie. Schloß Reisensburg (1984).

6 Jones. I. G.. M. N. Lowenthal, H. Buyst: Electrocardiographic changes in African trypanosomiasis caused by Trypanosoma brucei rhodesiense. Trans. roy. Soc. trop. Med. Hyg. 69 (1975). 388.

7 Knüttgen, H. J.: Zur Diagnostik und Therapie der perakuten Trypanosoma-rhodesiense-Infektionen mit hohen Parasitamien. In Gsell, O. (Hrsg.): Importierte Infektionskrankheiten. Epidemiologie und Therapie (Thieme: Stuttgart-New York 1980), 134

8 Mohr. W.: Zur Differentialdiagnose und Therapie eines Falles von Schlafkrankheit (Trypanosomiasis). Ther. d. Gegenw. 4 (1961), 227.

9 Pöhn. H.-P., R. Großmann: Malariaeinschleppungen 1980-1986 in die Bundesrepublik Deutschland einschließlich Berlin (West). Bundesgesundheitsblatt 31 (1988), 2.

10 Poltera. A. A.: Pathology of human African trypanosomiasis with reference to experimental African trypanosomiasis and infections of the central nervous system. Brit. med. Bull. 41 (1985). 169.

11 Recht, K., I. Pfurtner-Bloos, R. Gross: Importierte ostafrikanische Trypanosomiasis (Schlafkrankheit). Med. Welt 28 (1977). 1378.

12 Schechter, P. J., J. L. R. Barlow, A. Sjoerdsma: Clinical aspects; of inhibition of ornithin decarboxylase with emphasis on therapeutic trials of eflornithin (DFMO) in cancer and protozoan diseases. In McCann, P. P. (Ed.): Inhibition of Polyamine Metabolism (Academic Press: San Diego 1987). 345.

13 Statistisches Bundesamt: Fachserie 8. Reihe 6. Luftverkehr (Wiesbaden 1970-1987).

14 Steffen, B.: Reisemedizin. Epidemiologie der Gesundheitsstö. rungen bei Interkontinentalreisenden und praventivmedizinische Konsequenzen (Springer: Berlin-Heidelberg-New YorkTokyo 1984).

15 Sturchler. D.: Endemic Areas of Tropical Infections (Hans Huber: Toronto-Bern-Stuttgart 1988).

16 Werner. G. T.. H. Stickl: Tropenkrankheiten bei Auslandsreisenden. Fortschr. Med. 93 (1975). 561.

17 WHO: Epidemiology and control of African trypanosomiasis Wld Hith Org. techn. Rep. Ser. 739 (1986).

18 WHO: The primary health care approach to the control and prevention of sleeping sickness. Wkly epidem. Rec. 62 (1987). 197.

Dr. Th. Löscher. Dr. H. D. Nothdurft. Dr. F. von Sonnenburg Abteilung fur Infektions. und Tropenmedizin Medizinische Klinik Innenstadt der Universität Leopoldstr. 5 8000 München 40

Prof. Dr. H. Taelman

Institute of Tropical Medicine l.eopold II

Kronenburgstraat 43/3

B-2000 Antwerpen

Dr. M. Boogaerts. Dr. M. Omar Centre médico-social Kanombe Kigali. Ruanda 